\title{
NOTE ON CONVEX REGIONS ON THE SPHERE*
}

\section{R. M. ROBINSON}

By a convex region on the sphere we mean a region such that any great circle arc of length less than $180^{\circ}$, whose end points lie in the region, lies entirely in the region. Let $G$ denote any convex region, $G_{0}$ the diametrically opposite region, and $G_{1}$ the set obtained from the whole sphere by excluding $G$ and $G_{0}$ together with their boundaries. Let $\rho=\arctan \left(3^{1 / 2} / 2\right)$; then $40^{\circ} 53^{\prime}<\rho<40^{\circ} 54^{\prime}$. We shall prove the following theorem. $\dagger$

There is on the sphere a circle-interior of radius $\rho$ (measured on the sphere), which lies entirely in $G$ or entirely in $G_{1}$. The number $\rho$ cannot be replaced by any larger number.

Let $r$ be the least upper bound of the radii of circle-interiors lying in $G$. Then for every integer $n>1$ there is a circle-interior of radius $r(1-1 / n)$ lying in $G$. A limit point of their centers is the center of a circle-interior $C$ of radius $r$ lying in $G$. We may suppose that $G$ is neither the whole sphere nor a hemisphere, so that $r<90^{\circ}$.

No closed semicircumference forming part of the boundary of $C$ can be free of boundary points of $G$. For if it were; it would be at a distance $d>0$ from the boundary of $G$. Thus $G$ would contain not only $C$ but all points within a distance $d$ from one-half of its circumference. Hence $G$ would contain a circle-interior of radius greater than $r$.

If $P$ is any boundary point of $G$ on the circumference of $C$, then $G$ lies entirely on one side of the great circle tangent to $C$ at $P$. For if there were a point $P^{\prime}$ of $G$ on the opposite side of this great circle from $C$, we could join $P^{\prime}$ to $P$ by a great circle arc of less than $180^{\circ}$, which when extended through $P$ would cut $C$. Taking $P^{\prime \prime}$ as a point sufficiently near to $P$ on $P^{\prime} P$ extended, we should have $P^{\prime} P^{\prime \prime}<180^{\circ}$, $P$ on $P^{\prime} P^{\prime \prime}, P^{\prime}$ and $P^{\prime \prime}$ in $G$, therefore $P$ in $G$, contrary to hypothesis.

We shall show that there is an arc $P Q$ forming not more than onehalf nor less than one-third of the circumference of $C$, and whose end points $P$ and $Q$ are boundary points of $G$. Let $P_{1}$ be any boundary point of $G$ on the circumference of $C$, and let $P_{0}$ be the opposite point of the circumference. If $P_{0}$ is a boundary point of $G$, then we may take either arc $P_{1} P_{0}$ as $P Q$. Otherwise let $P_{2}$ and $P_{3}$ be the boundary

* Presented to the Society, November 27, 1937.

$\dagger$ This theorem was suggested to me by Hans Lewy, who makes use of it in a current paper. 
points of $G$ on the circumference of $C$ nearest to $P_{0}$ on either side. Then the $\operatorname{arcs} P_{1} P_{2}$ and $P_{1} P_{3}$ are less than a semicircumference by construction, and $P_{2} P_{3}$ cannot be greater than a semicircumference, since its interior is free of boundary points of $G$. The longest of the three arcs is at least one-third of the circumference, and hence can be taken as $P Q$.

Drawing the tangent great circles to $C$ at $P$ and $Q$, we have $G$ included within a lune, whose angle can be easily calculated (in terms of $r$ and the length of $P Q$ ). Let $2 \theta$ be the angle of the lune, and $2 \alpha$ the angle subtended by $P Q$ at the center of $C$. Then $60^{\circ} \leqq \alpha \leqq 90^{\circ}$. Join the nearer point of intersection of the tangents at $P$ and $Q$ to the center of $C$; also join $P$ to the center of $C$. A right triangle is formed with angles $\alpha$ and $\theta$, and side $r$ opposite the latter angle. Applying a formula true for any right spherical triangle, we have

$$
\cos \theta=\cos r \sin \alpha,
$$

hence $\cos \theta \geqq\left(3^{1 / 2} / 2\right) \cos r$. Let $r^{\prime}=90^{\circ}-\theta$. Then in either of the supplementary lunes there is a circle-interior of radius $r^{\prime}$, and

$$
\sin r^{\prime} \geqq\left(3^{1 / 2} / 2\right) \cos r .
$$

For values of $r$ and $r^{\prime}$ satisfying this inequality, the smallest value of $\max \left(r, r^{\prime}\right)$ will occur for $\sin r^{\prime}=\left(3^{1 / 2} / 2\right) \cos r$, and $r^{\prime}=r$. The common value of $r$ and $r^{\prime}$ is then $\rho=\arctan \left(3^{1 / 2} / 2\right)$. Hence in every case $\max \left(r, r^{\prime}\right) \geqq \rho$, so that there is always a circle-interior of radius $\rho$ in $G$ or $G_{1}$.

We must show that $\rho$ cannot be replaced by any larger number. For this purpose we find a particular region $G$ so that there is no circle-interior of radius greater than $\rho$ in $G$ or $G_{1}$. Let $G$ be the interior of an equilateral triangle circumscribed about a circle of radius $\rho$. If the angles of the triangle are $2 \theta$, we have (putting $r=\rho$ and $\alpha=60^{\circ}$ ) $\cos \theta=\left(3^{1 / 2} / 2\right) \cos \rho=\sin \rho$, or $\theta=90^{\circ}-\rho$. The angles of the triangle being more than $90^{\circ}$, the sides are also more than $90^{\circ}$. The exterior angles of the triangle are $2 \rho$. In $G_{1}$ there are then six circles of radius $\rho$, touching one side of $G$ and one side of $G_{0}$. There are no larger circles in $G_{1}$. For every maximum circle, since it cannot touch $G$ and $G_{0}$ at three points, must touch them at two opposite points of the circle. This can happen only for the six circles mentioned and for the six circles which pass through a vertex of one triangle and are tangent at the mid-point of a side of the other. These latter circles are seen, however, not to furnish even a relative maximum.

University of California 